\title{
Function of Krüppel-like factor 2 in the shear stress-induced cell differentiation of endothelial progenitor cells to endothelial cells
}

\author{
HAI-RONG CHU*, YU-CONG SUN*, YU GAO, XIU-MEI GUAN, HONG YAN, \\ XIAO-DONG CUI, XIAO-YUN ZHANG, XIN LI, HONG LI and MIN CHENG
}

Medicine Research Center, Clinical Medical College, Weifang Medical University, Weifang, Shandong 261053, P.R. China

Received February 26, 2018; Accepted October 15, 2018

DOI: $10.3892 / \mathrm{mmr} .2019 .9819$

\begin{abstract}
The present study aimed to evaluate the effects of Krüppel-like factor 2 (KLF2) on the differentiation of endothelial progenitor cells (EPCs) to endothelial cells (ECs) induced by shear stress, and to investigate the corresponding mechanisms. Cultured rat late EPCs were exposed to shear stress $\left(12 \mathrm{dyn} / \mathrm{cm}^{2}\right)$ for different lengths of time. Reverse transcription-quantitative polymerase chain reaction (RT-qPCR) was used to measure the initial KLF2 mRNA levels in each group. Subsequently, the EPCs were treated with anti-integrin $\beta 1$ or $\beta 3$ antibodies to block integrin $\beta 1$ and $\beta 3$, respectively, or cytochalasin D to destroy F-actin, and the subsequent expression levels of KLF2 in EPCs were measured. Then, KLF2 small interfering RNAs (siRNAs) were transfected into EPCs, and RT-qPCR was used to measure the mRNA expression level of KLF2. Additionally, flow cytometry was applied to evaluate the protein levels of cluster of differentiation 31 (CD31) and the von Willebrand factor (vWF), and the regulatory effects of KLF2 in the promoter region of $\mathrm{vWF}$ were determined via a luciferase assay. High shear stress upregulated KLF2 expression, while blocking integrin $\beta 1 / \beta 3$ or destroying $\mathrm{F}$-actin resulted in a corresponding decrease in KLF2 expression. Downregulation of KLF2 expression by siKLF2 inhibited the differentiation of EPCs to ECs under shear stress conditions, while the expression of EC-specific markers decreased, including CD31 and vWF. Various lengths of the vWF promoter region induced vWF expression, and EPCs co-transfected with KLF2 significantly increased the vWF expression levels compared with the group treated
\end{abstract}

Correspondence to: Professor Hong Li or Professor Min Cheng, Medicine Research Center, Clinical Medical College, Weifang Medical University, 7166 Baotongxi Road, Weicheng, Weifang, Shandong 261053, P.R. China

E-mail: mincheng@wfmc.edu.cn

E-mail:1h@wfmc.edu.cn

*Contributed equally

Key words: shear stress, endothelial progenitor cells, Krüppel-like factor 2, differentiation, endothelial cells with vWF alone $(\mathrm{P}<0.01)$. In conclusion, shear stress may upregulate KLF2 expression, which may be associated with the integrin-actin cytoskeleton system. Most importantly, the shear stress-induced differentiation of EPCs may be mediated by KLF2.

\section{Introduction}

Endothelial injury is the first step in the development of atherosclerosis (1). Numerous factors are involved in this endothelial injury process; for example, endothelial progenitor cells (EPCs) serve an important function in the maintenance of endothelium structural integrity and function $(2,3)$. Previous studies have revealed that shear stress promotes the differentiation of EPCs to endothelial cells (ECs), and participates in vascular re-endothelialization (4-6). However, the transcriptional regulatory mechanisms involved in the control of the differentiation of EPCs remain unclear.

Krüppel-like factor 2 (KLF2), a member of the zinc finger transcription factor family, regulates cellular growth during tissue development (7). Previously, KLF2 has emerged as a primary regulator of endothelial quiescence, anti-inflammatory responses, antithrombotic functions and vascular tone by activating atheroprotective transcription and inhibiting atherogenic transcription $(8,9)$. In addition, KLF2 is involved in the regulation of various immune cells by inhibiting the proinflammatory activation of monocytes (10). Das et al (11) revealed that KLF2 mediates the transcriptional regulation of arthritis via the modulation of monocyte differentiation and function. KLF2 expression is elevated in the vascular endothelium and required for normal vessel development $(12,13)$. In addition, it is a prominent anti-angiogenic factor, and modulates the expression of multiple endothelial vasoprotective genes (14). In previous years, numerous studies have revealed that KLF2 is also an important regulator for cardiovascular cells, particularly cardiac ECs. For example, Stroncek et al (15) demonstrated that KLF2 expression was substantially higher in the EPCs of patients with coronary artery disease compared with those of healthy individuals. KLF2 also controls the phenotype and metabolism of ECs (16). However, the function of KLF2 in the differentiation from EPCs to ECs remains unknown.

In the present study, the effects of KLF2 on the differentiation of EPCs to ECs under shear stress were investigated, in addition to the underlying mechanisms. One previous study 
demonstrated that shear stress may induce differentiation of EPCs to ECs in a magnitude-dependent manner through its effects on the integrin-actin cytoskeleton system, and may result in the increased expression of von Willebrand factor (vWF) and cluster of differentiation 31 (CD31) under a shear stress of $12 \mathrm{dyn} / \mathrm{cm}^{2}(5)$. However, the underlying transcriptional mechanism remains unclear. Therefore, the effects of KLF2 on the differentiation of EPCs to ECs were investigated through the expression of two EC-specific markers, including CD31 and vWF. These results may provide novel evidence for the function of KLF2 in EPC differentiation, and may illustrate the potential association between KLF2 and atherosclerosis-associated diseases.

\section{Materials and methods}

EPC culture and identification. The present study was conducted in accordance with the Guide for the Care and Use of Laboratory Animals published by the National Institutes of Health (17), and the protocol was ethically approved by the Local Animal Ethics Committee of Weifang Medical College (Weifang, China). Late EPCs were isolated and cultured as described previously (6). Briefly, bone marrow mononuclear cells (MNCs) were fractionated at $4^{\circ} \mathrm{C}$ with $700 \mathrm{x}$ g for $20 \mathrm{~min}$ by density gradient centrifugation with Histopaque $^{\circledR}-1083$ (Sigma-Aldrich; Merck KGaA, Darmstadt, Germany) from the whole bone marrow of Sprague-Dawley rats (n=10; weight, 150-175 g; age 8 weeks; Weifang Medical College, Weifang, China); rats were housed in controlled conditions: 12 -h light/dark cycle, $22^{\circ} \mathrm{C}, 60 \%$ humidity with $\mathrm{ad}$ libitum access to food. MNCs were plated on dishes precoated with $50 \mu \mathrm{g} / \mathrm{ml}$ fibronectin (Sigma-Aldrich; Merck KGaA), and were maintained at $37^{\circ} \mathrm{C}$ in complete EC growth medium-2 supplemented with $10 \%$ fetal calf serum (Invitrogen; Thermo Fisher Scientific, Inc., Waltham, MA, USA). Unattached cells were removed with sterile phosphate buffered saline (PBS) after 4 days, and endothelial colonies appeared subsequently. Late EPCs were used in the following experiments subsequent to 3-5 passages.

Cell treatments. To verify whether integrin $\beta 1 / \beta 3$ or F-actin influences the expression of KLF2 under shear stress, late EPCs in the experimental group were first pretreated with $50 \mu \mathrm{g} / \mathrm{ml}$ anti-integrin $\beta 1$ (clone MEM-101A; catalog no. CSB-PA011880LA01MO-100; Dianova GmbH, Hamburg, Germany) and $10 \mu \mathrm{g} / \mathrm{ml}$ anti-integrin $\beta 3$ (catalog no. MAB2023Z; Gibco; Merck KGaA) antibodies for $30 \mathrm{~min}$ at $37^{\circ} \mathrm{C}$, or incubated with $1 \mu \mathrm{mol} / 1$ cytochalasin D (cytoD; catalog no. PHZ1063; Thermo Fisher Scientific, Inc.) for $1 \mathrm{~h}$ at $37^{\circ} \mathrm{C}$ to disrupt actin filaments (6), as described previously (5). EPCs treated with dimethyl sulfoxide (DMSO) were used as the control group. Then, the two groups of EPCs were exposed to $12 \mathrm{dyn} / \mathrm{cm}^{2}$ shear stress for $1 \mathrm{~h}$. The KLF2 mRNA levels in the two groups were measured using reverse transcription-quantitative polymerase chain reaction (RT-qPCR) as described below.

Small interfering RNA (siRNA) was used to demonstrate the function of KLF2 under shear stress. KLF2 siRNAs $(20 \mu \mathrm{mol} / \mathrm{l})$ were transfected into EPCs $\left(10^{5}\right.$ cells $)$ using Lipofectamine ${ }^{\circledR} 3000$ (Invitrogen; Thermo Fisher Scientific,
Inc.) at room temperature, according to the manufacturer's protocol; the medium contained was changed after $6 \mathrm{~h}$ incubation. The cells were collected $72 \mathrm{~h}$ later, and the KLF2 mRNA expression levels were assessed to verify the efficiency of interference, while the expression levels of CD31 and vWF were determined using flow cytometry. The sequences for KLF2 siRNA were as follows: Sense, 5'-CAG GUGAGAAGCCUUAUCATT-3'; antisense, 5'-UGAUAA GGCUUCUCACCUGTT-3' (Shanghai GenePharma Co., Ltd., Shanghai, China). In addition, a non-targeting control was used as a negative control in the present study. The sequences of the negative control were as follows: Sense, 5'-UUCUCCGAACGUGUCACGUTT-3'; antisense, 3'-ACG UGACACGUUCGGAGAATT-5' (Shanghai GenePharma Co., Ltd.). Additionally, another group of EPCs transfected with si-KLF2 for $60 \mathrm{~h}$ were exposed to $12 \mathrm{dyn} / \mathrm{cm}^{2}$ shear stress for an additional $12 \mathrm{~h}$, and the expression levels of CD31 and vWF were analyzed using flow cytometry. The regulatory effects of KLF2 in the promoter region of $\mathrm{vWF}$ were determined via a luciferase assay.

Shear stress experiments. EPCs were subjected to shear stress using a flow chamber system as previously described (6). The following formula was used to calculate the stress intensity: $\tau_{\omega}=\frac{6 u}{h^{2} \mathrm{~b}} \mathrm{Q}$, where $\tau_{\omega}$ is the shear stress, $\mu$ the medium viscosity $(0.0077 \mathrm{~g} / \mathrm{cmNs}), \mathrm{Q}$ the volumetric flow rate $\left(2.05 \mathrm{~cm}^{3} / \mathrm{s}\right), \mathrm{h}$ the chamber height $(0.03 \mathrm{~cm})$ and $\mathrm{b}$ the chamber width $(2.5 \mathrm{~cm})$.

$R T-q P C R$. Total RNA was isolated from the aforementioned treated cells with TRIzol ${ }^{\circledR}$ reagent (Invitrogen; Thermo Fisher Scientific, Inc.), and the RNA was reverse-transcribed using a SYBR PrimeScript RT-PCR kit (Takara Bio, Inc., Otsu, Japan) at $37^{\circ} \mathrm{C}$ for $15 \mathrm{~min}$, according to the manufacture's protocol. RT-qPCR was performed using SYBR Premix Ex Taq (Takara Bio, Inc.) to determine gene expression levels. GAPDH was used as the internal control. The primer sequences were as follows: GAPDH, forward 5'-GGC ACAGTCAAGGCTGAGAATG-3', reverse 5'-ATGGTG GTGAAGACGCCAGTA-3'; KLF2, forward 5'-TCGCAC CTAAAGGCGCATC-3', reverse 5'-TAGTGGCGGGTA AGCTCGTC-3'; CD31, forward 5'-GACAGCCAAGGCAGA TGCAC-3', reverse 5'-ATTGGATGGCTTGGCCTGAA-3'; vWF, forward 5'-GCGTGGCAGTGGTAGAGT A-3', reverse 5'-GGAGATAGCGGGTGAAATA-3'. qPCR reactions were performed on a LightCycler 480II instrument (Roche Diagnostics, Indianapolis, IN, USA) with a final primer concentration of $0.4 \mu \mathrm{mol} / 1$ according to the manufacturer's protocol. Thermocycling conditions were as follows: $95^{\circ} \mathrm{C}$ for $30 \mathrm{sec}$; followed by 40 cycles at $95^{\circ} \mathrm{C}$ for $15 \mathrm{sec} ; 59^{\circ} \mathrm{C}$ for $1 \mathrm{~min}$; and $72^{\circ} \mathrm{C}$ for $10 \mathrm{sec}$. The fold changes in gene expression were calculated using the following formula: Fold change $=2^{-\Delta \Delta \mathrm{Cq}}(18)$.

Western blot analysis. EPCs were lysed at $4^{\circ} \mathrm{C}$ for $30 \mathrm{~min}$ in Radioimmunoprecipitation Assay lysis buffer (catalog no. C1053; Applygen Technologies Inc., Beijing, China), and protein concentrations were determined with the Pierce Bicinchoninic Acid Assay kit (Thermo Fisher Scientific, Inc.). An equal amount of proteins (40 $\mu \mathrm{g} /$ lane) were separated by $12 \%$ (wt/vol) SDS-PAGE, and the proteins were subsequently 
A

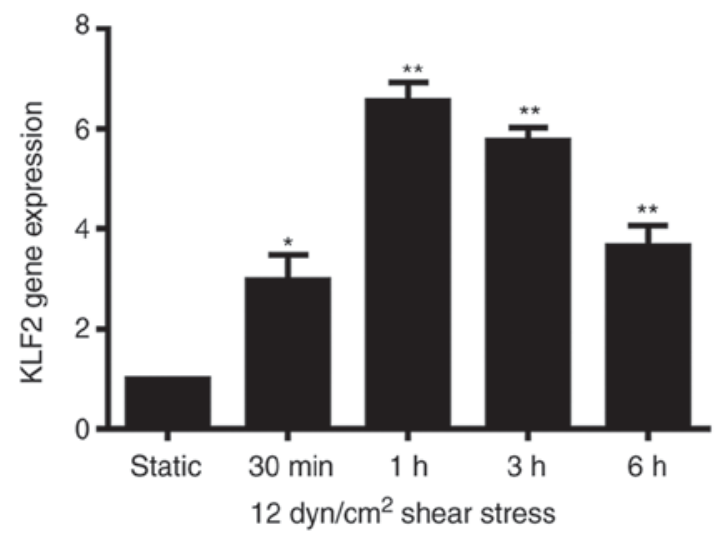

C

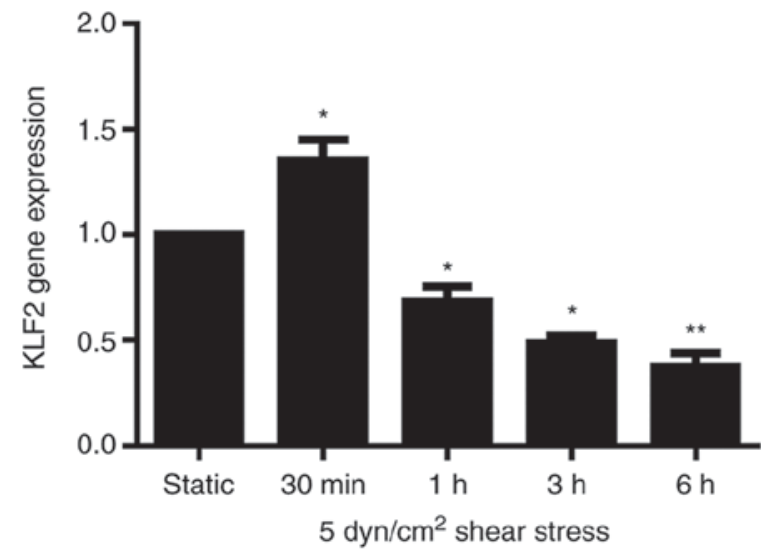

B
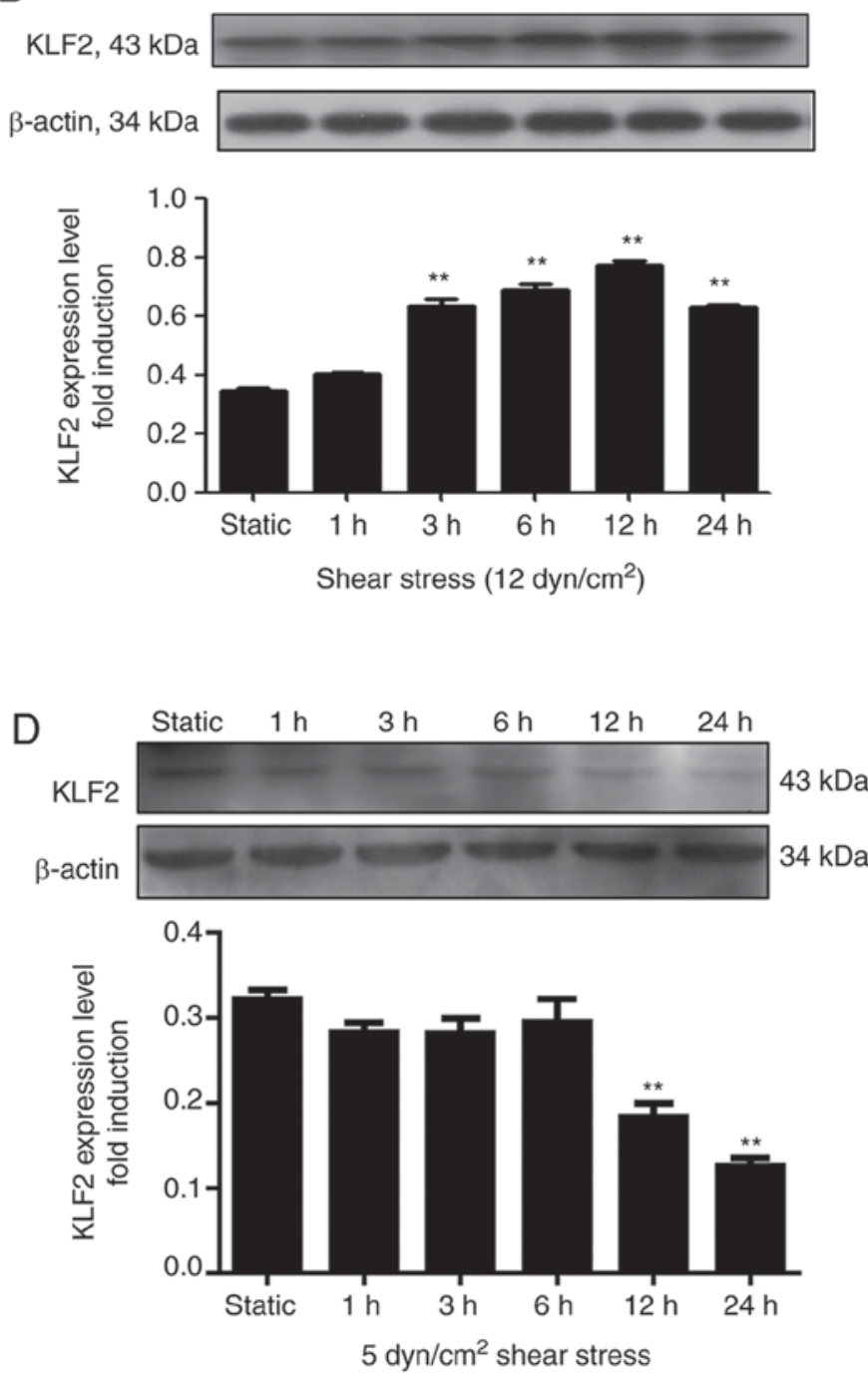

Figure 1. mRNA and protein expression levels of KLF2 in EPCs are affected by shear stress. (A and B) EPCs were maintained in static conditions or exposed to $12 \mathrm{dyn} / \mathrm{cm}^{2}$ laminar shear stress for varying lengths of time; (A) RT-qPCR analysis at static, $0.5,1,2,3$ and $6 \mathrm{~h}$, and (B) western blot analysis at static, 1, 3, 6 , 12 and $24 \mathrm{~h}$ revealed that KLF2 mRNA and protein expression levels, respectively, increased in EPCs following exposure to 12 dyn/cm ${ }^{2}$ shear stress for various time durations. (C and D) EPCs were also maintained in static conditions or exposed to 5 dyn $/ \mathrm{cm}^{2}$ laminar shear stress for varying lengths of time; (C) RT-qPCR analysis at static, 0.5, 1,3 and $6 \mathrm{~h}$ and (D) western blot analysis static, 1, 3, 6, 12 and $24 \mathrm{~h}$ revealed that KLF2 mRNA and protein expression levels, respectively, were reduced in EPCs subsequent to exposure to $5 \mathrm{dyn} / \mathrm{cm}^{2}$ shear stress for various time durations. Data were expressed as means \pm standard deviation from 4 independent experiments; $\mathrm{n}=4$; ${ }^{*} \mathrm{P}<0.05$ and ${ }^{* *} \mathrm{P}<0.01$ vs. Static group. EPCs, endothelial progenitor cells; KLF2, Krüppel-like factor 2; RT-qPCR, reverse transcription-quantitative polymerase chain reaction.

transferred to polyvinylidene fluoride membranes and blocked with $5 \%$ bovine serum albumin (catalog no. ST-023; Beyotime Institute of Biotechnology, Jiangsu, China ) in TBS with $0.05 \%$ Tween 20 (TBST) for $60 \mathrm{~min}$ at room temperature, followed by a $4^{\circ} \mathrm{C}$ overnight incubation with the primary antibodies against KLF2 (1:500; catalog no. SAB2108684; Sigma-Aldrich; Merck KGaA). $\beta$-actin (1:1,000; catalog no. AF0003; Beyotime Institute of Biotechnology) was used as a loading control and for normalization. Membranes were then washed with TBST and incubated with horseradish peroxidase (HRP)-conjugated mouse anti-rabbit immunoglobulin (Ig)G (1:1,000; SC-2357; Santa Cruz Biotechnology, Inc., Dallas, TX, USA) or HRP-conjugated goat anti-mouse $\operatorname{IgG}(\mathrm{H}+\mathrm{L})(1: 2,000$; catalog no. A0216; Beyotime Institute of Biotechnology) at room temperature for $60 \mathrm{~min}$. Immunoreactive bands were visualized using enhanced chemiluminescence (GE Healthcare,
Chicago, IL, USA) and densitometric analysis was conducted using ImageJ v1.8.0 software (National Institutes of Health, Bethesda, MD, USA).

Flow cytometry. Flow cytometry was used to determine the expression levels of CD31 and vWF. EPCs were trypsinized into a single cell suspension ( $1 \times 10^{5}$ cells), fixed with $4 \%$ paraformaldehyde for $15 \mathrm{~min}$ and washed three times with PBS for 5 min. Cells were permeabilized with $0.1 \%$ TritonX-100 for $5 \mathrm{~min}$, washed with PBS and incubated with phycoerythrin-conjugated anti-CD31 (1:50; catalog no. 25-0319-41; eBioscience; Thermo Fisher Scientific, Inc. ) or fluorescein isothiocyanate-conjugated anti-vWF (1:50; catalog no. ab8822; Abcam) for $1 \mathrm{~h}$ at $4^{\circ} \mathrm{C}$. Subsequent to washing with PBS to remove unbound antibodies, cells were analyzed using a FACSCanto II flow cytometer (BD Biosciences, San Jose, CA, 
USA) and the data were analyzed utilizing a software FlowJo 10 (Tree Star, Inc., Ashland, OR, USA).

Luciferase assay. For the luciferase assay, various lengths (1/4, $2 / 4,3 / 4$ and full length) of the 5'untranslated (5'UTR) region of vWF, including nucleotide regions 4851-5341 (490 bp), 4241-5341 (1,100 bp), 3848-5341 (1,493 bp) and 3341-5341 (2,000 bp), and KLF2 gene were inserted into a psiCheck2 vector, respectively (Promega Corporation, Madison, WI, USA). 293 cells (Type Culture Collection of the Chinese Academy of Sciences, Shanghai, China) were maintained in DMEM with $10 \%$ fetal bovine serum at $37^{\circ} \mathrm{C} .293$ cells, which express vWF, but not KLF2, were co-transfected with the KLF2-containing psiCheck2 plasmid using GeneJuice Transfection Reagent (Merck KGaA) at $37^{\circ} \mathrm{C}$ for $48 \mathrm{~h}$. Cells transfected with the unmodified psiCheck 2 empty vector were used as controls. Luciferase reporter gene activity was measured $48 \mathrm{~h}$ following transfection using the Promega Dual-luciferase reporter assay system (Promega Corporation) an OPtocomp I luminometer (MGM Instruments, Inc., Hamden, CT, USA).

Statistical analysis. SPSS software version 16.0 (SPSS, Inc., Chicago, IL, USA) was used to analyze all data. Unless otherwise indicated, the data were expressed as the mean \pm standard deviation. Statistical analyses were performed using one-way analysis of variance, followed by Tukey-Kramer multiple comparisons post-hoc tests. $\mathrm{P}<0.05$ was considered to indicate a statistically significant difference.

\section{Results}

KLF2 expression increases in response to shear stress. The KLF2 mRNA levels significantly increased in response to $12 \mathrm{dyn} / \mathrm{cm}^{2}$ laminar shear stress compared with the static group, peaking after $1 \mathrm{~h}(\mathrm{P}<0.01)$, and subsequently dropped, despite remaining significantly high compared with the static group at 3 and $6 \mathrm{~h}(\mathrm{P}<0.01$; Fig. 1A). The KLF2 protein expression level also demonstrated a significant increase in response to $3 \mathrm{~h}$ or more of $12 \mathrm{dyn} / \mathrm{cm}^{2}$ laminar shear stress compared with the static group $(\mathrm{P}<0.01)$, reaching a peak after $12 \mathrm{~h}$, and remaining comparatively high at $24 \mathrm{~h}$ (Fig. 1B). However, no significant differences were observed between the $1 \mathrm{~h}$ group and the static group. Furthermore, $5 \mathrm{dyn} / \mathrm{cm}^{2}$ shear stress inhibited KLF2 mRNA expression in a time-dependent manner when treated for $1 \mathrm{~h}$ or more (Fig. 1C), with significant differences identified between each group and the static group $(\mathrm{P}<0.05, \mathrm{P}<0.05$ and $\mathrm{P}<0.01$ for the 1,3 and $6 \mathrm{~h}$ groups, respectively); however, the $0.5 \mathrm{~h}$ group exhibited a significant increase in mRNA compared with the static group $(\mathrm{P}<0.05)$. It was speculated that the short duration $(<1 \mathrm{~h})$ of $5 \mathrm{dyn} / \mathrm{cm}^{2}$ shear stress treatment may have promoted KLF2 mRNA expression, and only longer durations $(>1 \mathrm{~h})$ of $5 \mathrm{dyn} / \mathrm{cm}^{2}$ shear stress treatment inhibited KLF2 mRNA expression in a time-dependent manner. Additionally, the KLF2 protein expression levels were significantly decreased at $12 \mathrm{~h}$ and $24 \mathrm{~h}$ of treatment, compared with the static group ( $\mathrm{P}<0.01$; Fig. 1D).

Expression of KLF2 decreases in response to treatment with anti-integrin $\beta 1, \beta 3$ and cytoD antibodies. KLF2 mRNA expression levels in the cytoD group were significantly
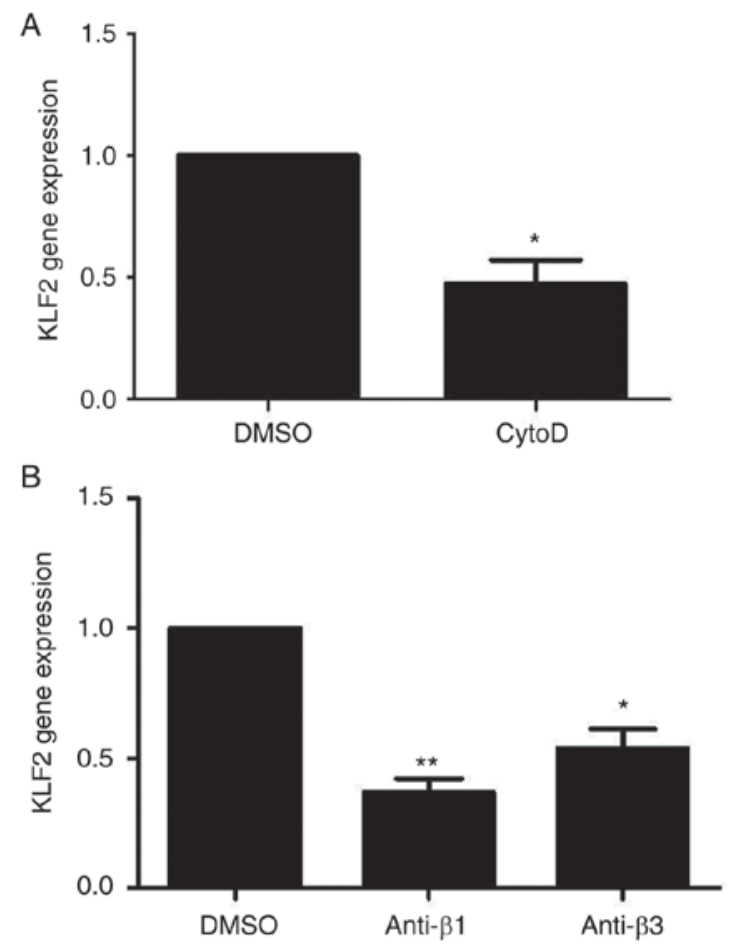

Figure 2. KLF2 expression decreases in EPCs treated with cytoD, anti-integrin $\beta 1$ or anti-integrin $\beta 3$ antibodies under shear stress conditions. (A and B) EPCs were pretreated with cytoD, anti-integrin $\beta 1$ or anti-integrin $\beta 3$ antibodies for $1 \mathrm{~h}$, then exposed to laminar shear stress $\left(12 \mathrm{dyn} / \mathrm{cm}^{2}\right)$ for $40 \mathrm{~min}$. Following pretreatment with (A) cytoD and (B) anti-integrin $\beta 1 / \beta 3$ antibodies, the mRNA expression levels of KLF2 decreased. GAPDH was used as an internal control; $\mathrm{n}=3 ;{ }^{*} \mathrm{P}<0.05$ and ${ }^{* *} \mathrm{P}<0.01$ vs. DMSO. cytoD, cytochalasin D; DMSO, dimethylsulfoxide; EPCs, endothelial progenitor cells; KLF2, Krüppel-like factor 2.

inhibited compared with the DMSO control group $(\mathrm{P}<0.05$; Fig. 2A). Similarly, the KLF2 gene expression levels in the anti-integrin $\beta 1(\mathrm{P}<0.01)$ and anti-integrin $\beta 3(\mathrm{P}<0.05)$ groups decreased significantly compared with the DMSO control group (Fig. 2B).

Expression levels of CD31 and $v W F$. In cells transfected with siKLF2, KLF2 mRNA expression levels decreased significantly compared with the control group $(\mathrm{P}<0.01 ;$ Fig. $3 \mathrm{~A})$. The mRNA levels of CD31 and vWF also decreased significantly compared with the control group (mRNA levels of CD31 vs. the control group $\mathrm{P}<0.05$; mRNA levels of vWF vs. the control group, $\mathrm{P}<0.01$; Fig. 3B). Additionally, results from flow cytometry revealed that the protein levels of CD31 and vWF increased significantly in response to shear stress, compared with the static group $(\mathrm{P}<0.01$; Fig. $3 \mathrm{C}$ and $\mathrm{D})$; however, siKLF2 pretreatment appeared to reverse this effect $(\mathrm{P}<0.05$; Fig. 3C and D).

KLF2 binds to the promoter region of $v W F$. Luciferase assay results revealed that various lengths of the vWF 5'UTR may serve a function in inducing gene expression; however, there were no statistical differences in the activity in the different lengths of the vWF 5'UTR. The result demonstrated that the length of the vWF 5'UTR was not related to the gene expression. In 293 cells co-transfected with KLF2, the expression level of vWF significantly increased compared with the vWF 
A
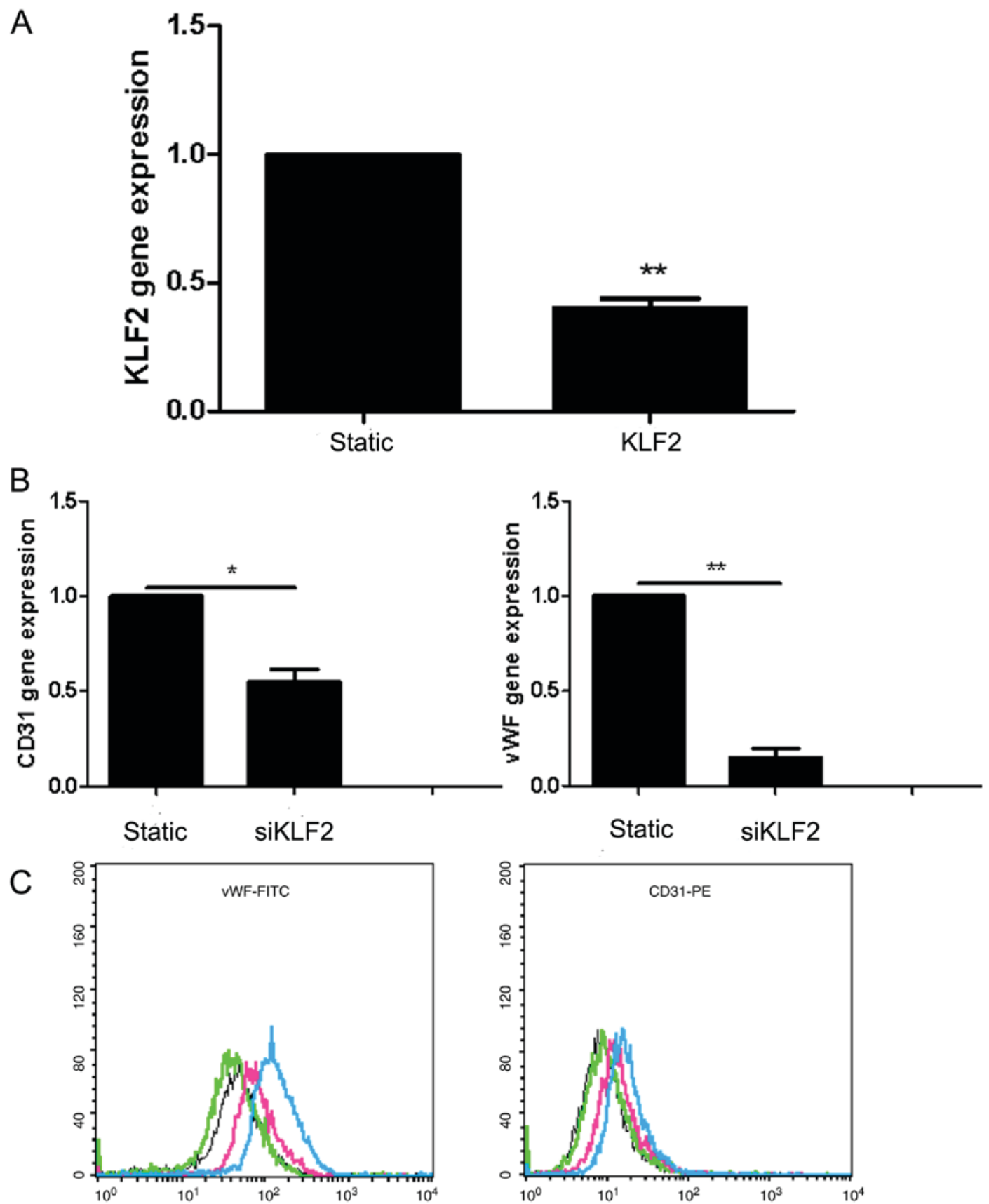

Static

siKLF2

D
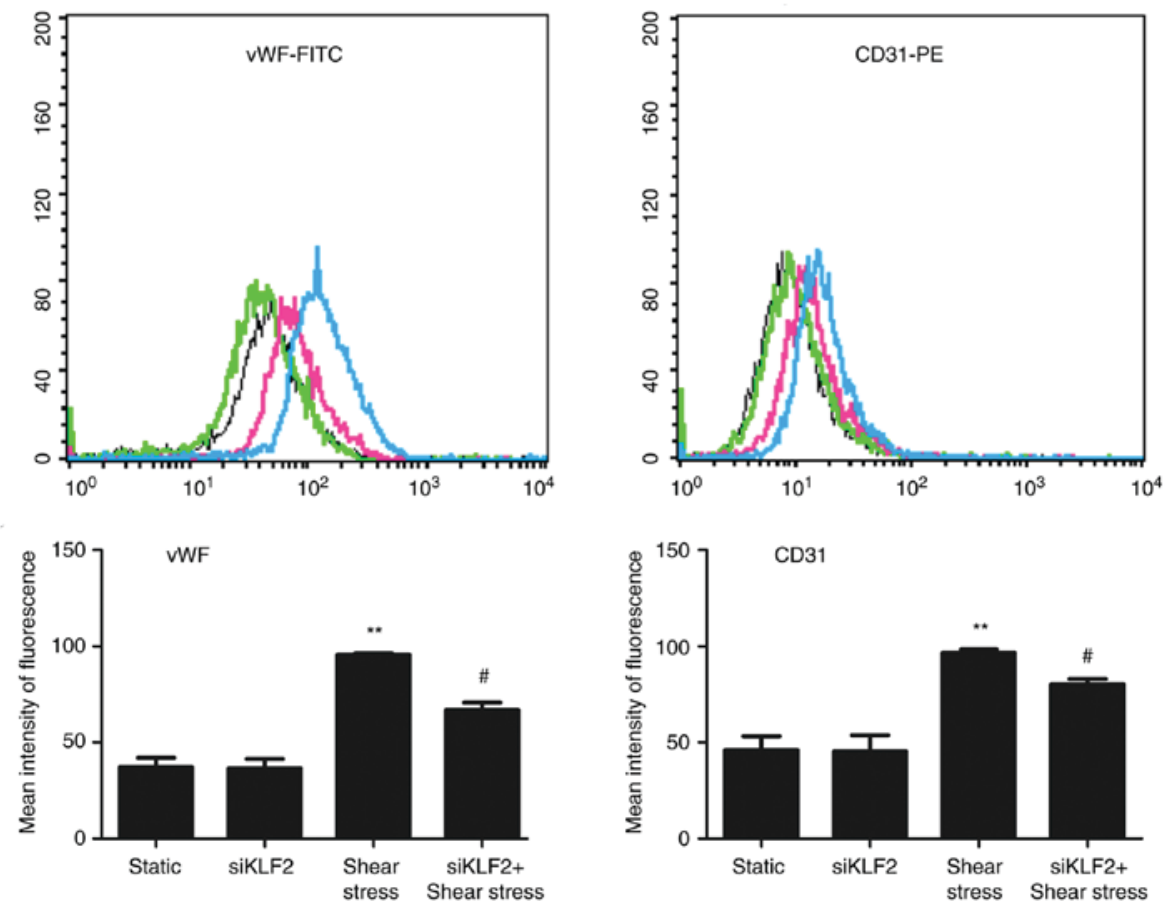

Figure 3. Effects of siKLF2 on the expression of EC markers in EPCs. (A-D) EPCs were treated with siKLF2 (20 $\mu \mathrm{M} / \mathrm{l})$ for $72 \mathrm{~h}$ under static conditions, and the expression levels of KLF2 and EC markers were determined using reverse transcription-quantitative polymerase chain reaction and flow cytometry, respectively. (A) siKLF2 transfection successfully decreased KLF2 mRNA expression levels; GAPDH was used to normalize gene expression; $\mathrm{n}=4$; * $\mathrm{P}<0.01$ vs. (B) siKLF2 transfection significantly decreased the mRNA expression levels of CD31 and vWF; ${ }^{*} \mathrm{P}<0.05,{ }^{* *} \mathrm{P}<0.01$. (C) Luciferase assay results revealed that siKLF2 transfection decreased protein expression levels of the EC makers vWF and CD31, under static and shear stress conditions. Black and green lines represent the static and the siKLF2-transfected groups, respectively; blue and red lines represent the shear stress and the siKLF2 + shear stress groups, respectively. (D) Quantification of luciferase assay results from Part C. siKLF2 + shear stress decreased protein expression level of EC marker. Values are expressed as the relative mean fluorescence intensity \pm standard deviation; $\mathrm{n}=3 ;{ }^{* * *} \mathrm{P}<0.01$ vs. Static group, ${ }^{*} \mathrm{P}<0.05$ vs. siKLF2 alone. EC, endothelial cells; EPCs, endothelial progenitor cells; FITC, fluorescein isothiocyanate; KLF2, Krüppel-like factor 2; PE, phycoerythrin; siRNA, small interfering RNA; vWF, von Willebrand factor. 


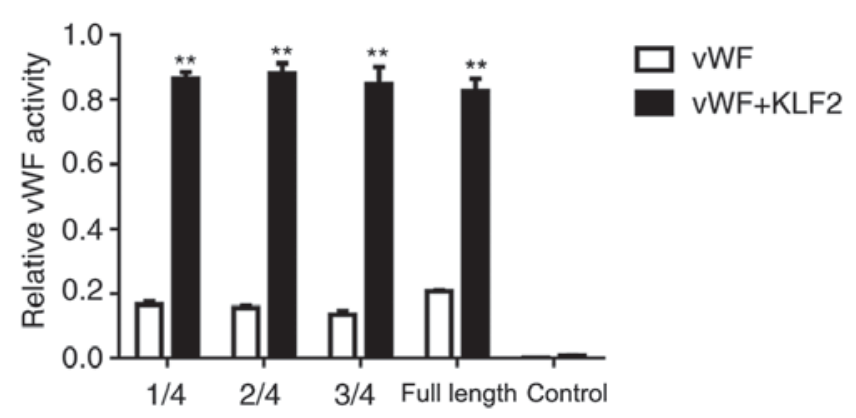

Figure 4. Luciferase assay of vWF expression in endothelial progenitor cells transfected with various lengths (1/4, 2/4, 3/4 and full length) of the vWF promoter, or co-transfected with KLF2 expression plasmid. The fluorescence values were significantly increased in the vWF+KLP2 groups compared with the respective vWF-only groups; ${ }^{* *} \mathrm{P}<0.01$ vs. vWF alone. KLF2, Krüppel-like factor 2; vWF, von Willebrand factor.

alone group ( $\mathrm{P}<0.01$; Fig. 4). KLF2 effectively bound to the vWF promoter, thus increasing the expression of vWF (as demonstrated by the increased fluorescence intensity) (Fig. 4).

\section{Discussion}

Shear stress serves an important function in the proliferation and differentiation of numerous types of cells, including ECs, stem cells and EPCs $(19,20)$. Mechanotransduction, which induces changes in endothelial metabolism, is a response to the extracellular stimuli of ECs and EPCs (21). The results of the present study revealed that shear stress upregulated the expression of KLF2. Downregulation of KLF2 expression by siKLF2 significantly inhibited the differentiation of EPCs to ECs under shear stress conditions. Additionally, KLF2 was involved in the regulation of vWF through binding to its promoter region.

Previous studies have demonstrated that KLF2, which may be induced by shear stress, contributes to maintaining a healthier endothelium by inducing a quiescent state, in addition to having antithrombotic, vasorelaxing and anti-inflammatory effects $(8,9)$. The results of these previous studies consistently revealed that shear stress increased KLF2 expression in late EPCs, particularly when treated with $12 \mathrm{dyn} / \mathrm{cm}^{2}$ shear stress for $1 \mathrm{~h}$, as applied in the present study. Results from a previous study revealed that KLF2 is a central transcriptional switch point, and is involved in the functional quiescent differentiation of the endothelium (9). Thus, it was hypothesized that KLF2 may regulate the differentiation of EPCs to ECs. To the best of our knowledge, there is no direct evidence regarding the effects of KLF2 on EPCs. Therefore, the present study investigated the potential mechanism underlying the effects of shear stress on KLF2 in EPCs. In the present study, KLF2 expression increased in response to relatively high shear stress $\left(12 \mathrm{dyn} / \mathrm{cm}^{2}\right)$, and decreased under relatively low shear stress $\left(5 \mathrm{dyn} / \mathrm{cm}^{2}\right)$, indicating that high shear stress may increase KLF2 expression. One previous study has revealed that shear stress of 6,12 and $20 \mathrm{dyn} / \mathrm{cm}^{2}$ substantially increased the expression of EC differentiation markers including $\mathrm{vWF}$ and CD31 in late EPCs, while no effect was observed at a stress level of $2 \mathrm{dyn} / \mathrm{cm}^{2}$. These results suggest that shear stress-induced EPC differentiation is associated with the intensity of shear stress (22). This is may be an explanation as to why atherosclerotic lesions occur predominantly in regions of the vasculature exposed to low shear stress (23). KLF2 transcription factor was revealed to be a protective mechanism for the endothelium against inflammatory and thrombotic events $(9,24)$. Additionally, a $12 \mathrm{dyn} / \mathrm{cm}^{2}$ laminar shear stress was also demonstrated to be a protective factor for the endothelium, while a $5 \mathrm{dyn} / \mathrm{cm}^{2}$ laminar shear stress may result in the formation of atherosclerosis $(25,26)$. These results suggest that KLF2 function in EPCs differentiation may be associated with the intensity of shear stress.

Boon et al (27) demonstrated that KLF2 was crucial to shear stress-mediated actin cytoskeleton remodeling and shear fiber assembly in the vascular endothelium. Furthermore, these fibers and forces may be essential for the firm attachment of ECs to withstand vascular mechanical forces. Additionally, microtubules may contribute to the cytoskeletal-dependent regulation of KLF2 expression (28). A similar trend in ECs, whose alignment was induced by fluid shear stress, further suggests a potential cytoskeletal-dependent regulation of KLF2 (28). Furthermore, previous studies have uncovered that shear stress may promote the differentiation of EPCs to ECs via the integrin-actin cytoskeleton system $(5,6)$. Accordingly, it was hypothesized that integrin $\beta 1 / \beta 3$ or F-actin may influence the expression of KLF2 under shear stress conditions. The results of the present study suggested that blocking integrin $\beta 1 / \beta 3$ or destroying actin fibers inhibited the expression of KLF2, suggesting that high shear stress may increase KLF2 expression through the integrin-actin cytoskeleton system. However, the inhibiting effects of low shear stress on KLF2 expression should be given greater attention, and further studies should be conducted on this topic.

EPCs participate in the process of endothelium repair through differentiation to ECS (29). To date, numerous studies have focused on the transcription factors active during EPC differentiation under static conditions (30-32). EPCs, which are located primarily in the bone marrow and peripheral blood, are transported to the injury area through blood circulation (33). Therefore, the effect of fluid shear stress may also be important for EPC differentiation. The regulatory effect of KLF2 on EC-specific markers, including CD31 and vWF, was noted in the static state and in the shear stress state in the present study. This suggests that KLF2 may serve an important function in the differentiation of EPCs to ECs under static and shear stress conditions, but particularly the latter. The present research revealed that KLF2 influenced EPC differentiation through binding to the promoter region of $\mathrm{vWF}$. This result is consistent with that of Hough et al (34) who argued that vWF promoter activity was enhanced 3.4-fold when ECs were exposed to shear stress. Furthermore, KLF2 bound to the vWF promoter effectively, thus increasing the fluorescence value during a luciferase assay, and no substantial differences were observed in vWF expression between the one-quarter length shortened promoter and the full-length promoter. This illustrated that there may be binding sites in this shortened promoter, but the specific loci remain unknown. However, there was a limitation in the present study, in that functional analysis of the EPCs with KLF2 knockdown was not performed, and that further studies are required to investigate the functional effects. 
In conclusion, the present study provides potential mechanistic insights into the promoting effects of KLF2 on EPC differentiation under shear stress conditions.

\section{Acknowledgements}

Not applicable.

\section{Funding}

The present study was supported by The National Natural Science Foundation of China (grant nos. 31570941, 31270993 and 81700406), The Program for New Century Excellent Talents in University (grant no. NCET-10-0922), The Natural Science Foundation of Shandong Province (grant nos. ZR2013CQ032, ZR2014JL018 and KX2014015), The Traditional Chinese Medicine of Shandong Province (grant nos. 2016WS0667 and 2015-239) and The Shandong Province Higher Educational Science and Technology Program (grant nos. J14LK59, J14LK12 and J15LK08).

\section{Availability of data and materials}

All data generated or analyzed during the present study are included in this published article.

\section{Authors' contributions}

$\mathrm{HC}$ and YS conceived and designed the research; YG, XG and HL performed the experiments and acquired the data; HY, XC and $\mathrm{MC}$ analyzed and interpreted the data; XZ and ZL performed statistical analyses; HC and YS drafted the manuscript; HL and $\mathrm{MC}$ revised the manuscript for important intellectual content. All authors read and approved the final manuscript.

\section{Ethics approval and consent to participate}

The present study was conducted in accordance with The Guide for the Care and Use of Laboratory Animals published by the National Institutes of Health (publication no. 85-23, revised 1996), and the protocol was approved by the Local Animal Ethics Committee of Weifang Medical College (Weifang, China).

\section{Patient consent for publication}

Not applicable.

\section{Competing interests}

The authors declare that they have no competing interests.

\section{References}

1. Rafieian-Kopaei M, Setorki M, Doudi M, Baradaran A and Nasri H: Atherosclerosis: Process, indicators, risk factors and new hopes. Int J Prev Med 5: 927-946, 2014.

2. Skrzypkowska M, Myśliwska J, Słomiński B, Siebert J, Gutknecht P and Rybastanisławowska M: Quantitative and functional characteristics of endothelial progenitor cells in newly diagnosed hypertensive patients. J Hum Hypertens 29: 324-330, 2015.
3. Stancu CS, Toma L and Sima AV: Dual role of lipoproteins in endothelial cell dysfunction in atherosclerosis. Cell Tissue Res 349: 433-446, 2012.

4. Zhang X, Cui X, Cheng L, Guan X, Li H, Li X and Cheng M: Actin stabilization by jasplakinolide affects the function of bone marrow-derived late endothelial progenitor cells. PLoS One 7: e50899, 2012

5. Cui X, Zhang X, Guan X, Li H, Li X, Lu H and Cheng M: Shear stress augments the endothelial cell differentiation marker expression in late EPCs by upregulating integrins. Biochem Biophys Res Commun 425: 419-425, 2012.

6. Cheng M, Guan X, Li H, Cui X, Zhang X, Li X, Jing X, Wu H and Avsar E: Shear stress regulates late EPC differentiation via mechanosensitive molecule-mediated cytoskeletal rearrangement. Plos One 8: e67675, 2013.

7. Bieker JJ: Kruppel-like factors: Three fingers in many pies. J Biol Chem 276: 34355-34358, 2001.

8. Dekker RJ, van Thienen JV, Rohlena J, de Jager SC, Elderkamp YW, Seppen J, de Vries CJ, Biessen EA, van Berkel TJ, Pannekoek $\mathrm{H}$ and Horrevoets AJ: Endothelial KLF2 links local arterial shear stress levels to the expression of vascular tone-regulating genes. Am J Pathol 167: 609-618, 2005.

9. Dekker RJ, Boon RA, Rondaij MG, Kragt A, Volger OL, Elderkamp YW, Meijers JC, Voorberg J, Pannekoek H and Horrevoets AJ: KLF2 provokes a gene expression pattern that establishes functional quiescent differentiation of the endothelium. Blood 107: 4354-5363, 2006.

10. He S, Yang L, Li D and Li M: Kruppel-like factor 2-mediated suppression of MicroRNA-155 reduces the proinflammatory activation of macrophages. Plos One 10: e139060, 2015.

11. Das M, Lu J, Joseph M, Aggarwal R, Kanji S, McMichael BK, Lee BS, Agarwal S, Ray-Chaudhury A, Iwenofu OH, et al: Kruppel-like factor 2 (KLF2) regulates monocyte differentiation and functions in mBSA and il-1 $\beta$-induced arthritis. Curr Mol Med 12: 113-125, 2012.

12. Anderson KP, Kern CB, Crable SC and Lingrel JB: Isolation of a gene encoding a functional zinc finger protein homologous to erythroid Krüppel-like factor: Identification of a new multigene family. Mol Cell Biol 15: 5957-5965, 1995.

13. Kuo CT, Veselits ML, Barton KP, Lu MM, Clendenin C and Leiden JM: The LKLF transcription factor is required for normal tunica media formation and blood vessel stabilization during murine embryogenesis. Genes Dev 11: 2996-3006, 1997.

14. Taniguchi H, Jacinto FV, Villanueva A, Fernandez AF Yamamoto H, Carmona FJ, Puertas S, Marquez VE, Shinomura Y, Imai K and Esteller M: Silencing of Kruppel-like factor 2 by the histone methyltransferase EZH2 in human cancer. Oncogene 31: 1988-1994, 2012.

15. Stroncek JD, Grant BS, Brown MA, Povsic TJ, Truskey GA and Reichert WM: Comparison of endothelial cell phenotypic markers of late-outgrowth endothelial progenitor cells isolated from patients with coronary artery disease and healthy volunteers. Tissue Eng Part A 15: 3473-3486, 2009.

16. Doddaballapur A, Michalik KM, Manavski Y, Lucas T, Houtkooper RH, You X, Chen W, Zeiher AM, Potente M, Dimmeler S and Boon RA: Laminar shear stress inhibits endothelial cell metabolism via KLF2-mediated repression of PFKFB3. Arterioscler Thromb Vasc Biol 35: 137-145, 2015.

17. National Research Council: Guide for the Care and Use of Laboratory Animals. The National Academies Press, Washington, DC, 1996.

18. Livak KJ and Schmittgen TD: Analysis of relative gene expression data using real-time quantitative PCR and the 2(-Delta Delta C(T)) method. Methods 25: 402-408, 2001

19. Obi S, Masuda H, Shizuno T, Sato A, Yamamoto K, Ando J, Abe Y and Asahara T: Fluid shear stress induces differentiation of circulating phenotype endothelial progenitor cells. Am J Physiol Cell Physiol 303: C595-C606, 2012.

20. Ahsan T and Nerem RM: Fluid shear stress promotes an endothelial-like phenotype during the early differentiation of embryonic stem cells. Tissue Eng Part A 16: 3547-3553, 2010.

21. Chien S: Mechanotransduction and endothelial cell homeostasis: The wisdom of the cell. Am J Physiol Heart Circ Physiol 292: H1209-H1224, 2007.

22. Ehsani Ardakani MJ, Safaei A, Arefi Oskouie A, Haghparast H, Haghazali M, Mohaghegh Shalmani H, Pey vandi H, Naderi N and Zali MR: Evaluation of liver cirrhosis and hepatocellular carcinoma using protein-protein interaction networks. Gastroenterol Hepatol Bed Bench 9 (Suppl 1): S14-S22, 2016. 
23. Cecchi E, Giglioli C, Valente S, Lazzeri C, Gensini GF, Abbate R and Mannini L: Role of hemodynamic shear stress in cardiovascular disease. Atherosclerosis 214: 249-256, 2011.

24. Doddaballapur A, Michalik KM, Manavski Y, Lucas T, Houtkooper RH, You X, Chen W, Zeiher AM, Potente M, Dimmeler $\mathrm{S}$ and Boon RA: Laminar shear stress inhibits endothelial cell metabolism via KLF2-mediated repression of PFKFB3. Arterioscler Thromb Vasc Biol 35: 137-145, 2015.

25. Lehoux S and Jones EA: Shear stress, arterial identity and atherosclerosis. Thromb Haemost 115: 467-473, 2016.

26. Olivon VC, Fraga-Silva RA, Segers D, Demougeot C, de Oliveira AM, Savergnini SS, Berthelot A, de Crom R, Krams R, Stergiopulos N and da Silva RF: Arginase inhibition prevents the low shear stress-induced development of vulnerable atherosclerotic plaques in ApoE-/- mice. Atherosclerosis 227: 236-243, 2013.

27. Boon RA, Leyen TA, Fontijn RD, Fledderus JO, Baggen JM, Volger OL, van Nieuw Amerongen GP and Horrevoets AJ: KLF2-induced actin shear fibers control both alignment to flow and JNK signaling in vascular endothelium. Blood 115: 2533-2542, 2010.

28. Vartanian KB, Berny MA, Mccarty OJ, Hanson SR and Hinds MT: Cytoskeletal structure regulates endothelial cell immunogenicity independent of fluid shear stress. Am J Physiol Cell Physiol 298: C333-C341, 2010.
29. Lu C, Zhang J, Zhang D, Uzan G and Li M: EPCs in vascular repair: How can we clear the hurdles between bench and bedside? Front Biosci (Landmark Ed) 19: 34-48, 2014.

30. Li X, Song Y, Wang D, Fu C, Zhu Z, Han Y, Li C, Wang N and Zhu Y: LIF maintains progenitor phenotype of endothelial progenitor cells via Krüppel-like factor 4. Microvasc Res 84: 270-277, 2012.

31. Cheng BB, Qu MJ, Wu LL, Shen Y, Yan ZQ, Zhang P, Qi YX and Jiang ZL: MicroRNA-34a targets Forkhead box j2 to modulate differentiation of endothelial progenitor cells in response to shear stress. J Mol Cell Cardiol 74: 4-12, 2014.

32. Wei S, Huang J, Li Y, Zhao J, Luo Y, Meng X, Sun H, Zhou X, Zhang $\mathrm{M}$ and Zhang W: Novel zinc finger transcription factor ZFP580 promotes differentiation of bone marrow-derived endothelial progenitor cells into endothelial cells via eNOS/NO pathway. J Mol Cell Cardiol 87: 17-26, 2015.

33. Takahashi T, Kalka C, Masuda H, Chen D, Silver M, Kearney M, Magner M, Isner JM and Asahara T: Ischemia- and cytokine-induced mobilization of bone marrow-derived endothelial progenitor cells for neovascularization. Nat Med 5: 434-438, 1999.

34. Hough C, Cameron CL, Notley CR, Brown C, O'Brien L, Keightley AM, Berber E and Lillicrap D: Influence of a GT repeat element on shear stress responsiveness of the VWF gene promoter. J Thromb Haemost 6: 1183-1190, 2008. 